\title{
ON ANOMALOUS DEPENDENCE OF CURRENT CARRIER CONCENTRATION ON THICKNESS IN THIN BISMUTH FILMS
}

\author{
G. Musial \\ Institute of Physics, Adam Mickiewicz University \\ Umultowska 85, 61-614 Poznan, Poland
}

\begin{abstract}
Using a simple model of thin film a dependence of the current carrier concentration $N$ on thickness $L$ in thin bismuth films is calculated in the conditions of the quantum size effect. Applying the parametrized form of the thin film potential it is shown that the experimentally determined anomalous dependence $N(L)$ can be obtained theoretically, assuming the standard boundary conditions in contrast to the results reported in literature. It is proved that the very structure of the hole energy spectrum is responsible for the anomalous character of the $N(L)$ dependence.
\end{abstract}

PACS numbers: 73.20.-r

It is well known that in the conditions of the quantum size effect (i.e. when a thickness of a film is comparable to the de Broglie wavelength of the conduction electrons) the theoretically calculated concentration of the current carriers $N_{\text {th }}$ increases with increasing thickness of the bismuth thin film (TF), under standard boundary conditions, that is zeroing of the carrier wave function at the TF surface (see Refs. [1-3] and papers cited therein). The same concentration $N_{\text {exp }}$ calculated from the experimental data decreases with increasing thickness of TF $[1,4]$. Trying to find a reason for this discrepancy the authors of Refs. [1,2] have assumed as the boundary conditions the zeroing of the gradient of the current carriers wave function on the TF surface. This condition allows the wave function to be nonzero anywhere outside the film. The concentration $N_{\text {th }}$ calculated according to the above decreases with increasing thickness of TF, but in both cases it is by one order of magnitude greater than $N_{\text {exp. }}$. An essential assumption taken by the above-mentioned authors is the quasicontinuous character of the hole energy spectrum, the same as in the bulk specimen. In Ref. [3] the energy spectrum of the current carriers was calculated assuming the vanishing-gradient-of-wave-function boundary conditions mentioned above and the energy-momentum relationship in the form known from the experimental data [5]. However, their result is valid for the temperature $0 \mathrm{~K}$ only.

Both physical situations described above are extreme. In this paper we calculate the electron concentration in thin bismuth film and we choose the standard 
boundary conditions in the parametrized form to eliminate the above-mentioned discrepancy between $N_{\text {exp }}$ and $N_{\text {th }}$ without any further artificial assumptions.

We solve the Schrödinger equation: $\left[\widehat{H}_{0}+V(x)\right] \psi(\boldsymbol{r})=E \psi(r)$, where $\widehat{H}_{0}$ is the current carrier Hamiltonian in an infinite medium and $V(x)$ is the current carrier potential energy connected with the presence of the TF surfaces (which are the surfaces $x= \pm d$ here). Therefore here the thickness of TF is $L=2 d$. We assume the simple model of TF according to which electrons and holes are considered as independent particles moving in the potential well $V(x)$. We expect that this model preserves the main features of a real TF. The electron Hamiltonian $\widehat{H}_{0}$ is of the form $\widehat{H}_{0}=\zeta_{i k}^{-1} \widehat{p}_{i} \widehat{p}_{k} / 2 m_{0}$, and the hole one $\widehat{H}_{0}=\eta_{i k}^{-1} \widehat{p}_{i} \hat{p}_{k} / 2 m_{0}$. In both last formulas we have applied the summation over the repeated indices, $m_{0}$ is the free electron mass. The elements of the inverse effective mass tensors $\zeta_{i k}^{-1}$ and $\eta_{i k}^{-1}$ have been calculated from the data from Ref. [5].

The cut off of the crystal lattice causes an increase in the current carriers potential energy at the TF surfaces, but it is hard to specify the exact form of the $V(x)$ dependence obtained on the basis of general principles. Therefore one should take the parametrized form of $V(x)$ to determine the detailed run of $V(x)$ in the vicinity of the surfaces. We take the following form of the potential energy

$$
\begin{aligned}
V(x) & =V_{0}\left\{1-\left(\arctan \frac{x+d}{a_{1}}-\arctan \frac{x-d}{a_{1}}\right) / \pi\right. \\
& \left.+w\left[1+\left(\arctan \frac{x+d-b}{a_{2}}-\arctan \frac{x-d+b}{a_{2}}\right) / \pi\right]\right\},
\end{aligned}
$$

where. $V_{0}, w, b, a_{1}, a_{2}$ are parameters. We can obtain the shape of $N_{\text {th }}(L)$ decreasing with increasing thickness of TF, as it was observed experimentally $[1,4]$, by variation of the values of these parameters. This form of $V(x)$ allows the wave function to be nonzero outside but only close to the film. Taking this form of $V(x)$ we introduce the possibility of existence of the potential pockets of width $b$ at the film surfaces, when $w \neq 0$, which is consistent with the qualitative considerations given in Ref. [6]. In fact we consider a sandwich made of a layer whose potential is practically constant surrounded by two layers whose potential decreases to 0 . The parameters $a_{1}$ and $a_{2}$ control the distance over which $V(x)$ changes from $V_{0}$ to 0 and 0 to $w V_{0}$ (the pocket depth), respectively. formula:

The number $s$ of particles in our system can be found from the following

$$
s=(\partial \Omega / \partial \mu)_{T, V},
$$

where $\Omega$ is the thermodynamical potential of the system, $\mu$ is the chemical potential, $V=2 d L_{y} L_{z}$ is the volume of the TF, $L_{y}$ and $L_{z}$ are the large sizes of the TF in the $y$ and $z$ directions parallel to the TF surface, $T$ is the temperature of the system assumed to be $4.2 \mathrm{~K}$. From Eq. (2) we can find the concentration of particles $N$, i.e. the number of particles per an unit of TF volume of the system

$$
N=\frac{1}{V} \sum_{n, p_{y}, p_{z}, \sigma} \exp \frac{\mu-E\left(n, p_{y}, p_{z}, \sigma\right)}{k_{\mathrm{B}} T} /\left[1+\exp \frac{\mu-E\left(n, p_{y}, p_{z}, \sigma\right)}{k_{\mathrm{B}} T}\right] .
$$


The equation of neutrality may be solved for $\mu_{\mathrm{e}}$ (or for $\mu_{\mathrm{h}}$ ) taking into regard the fact that $\mu_{\mathrm{e}}+\mu_{\mathrm{h}}=\Delta$, where $\Delta$ is the overlap of the electron and the hole band and equals to $61.6 \times 10^{-22} \mathrm{~J}$ [5]. Then one can obtain the electron concentration $N_{\mathrm{e}}$ from Eq. (3).

The results of our calculations show that there is no possibility to obtain $N_{\mathrm{e}}(L)$ decreasing with increasing thickness of TF for the standard boundary conditions, when $w=0$ (no potential pockets at the surfaces) for any values of the remaining potential parameters. Only the assumption of $w \neq 0$ provides the proper shape of the curve $N_{\mathrm{e}}(L)$ known from Refs. [1,4] which is illustrated in Fig. 1 . Moreover, the electron energy spectrum differs slightly from the spectrum for $w=0$ and from that obtained by the authors of Refs. [1,2] for the standard boundary conditions. At $w=0.015$ no electron level lies below the value $w V_{0}$, i.e. in the potential pocket region, because of the small masses of electrons in bismuth. The basic difference is the shape of the hole energy spectrum. A large mass of holes in bismuth is responsible for the fact that in the potential pocket region (i.e. below the value $w V_{0}$ ) there are two twice degenerated levels whose energy remains constant in the considered region of thickness.

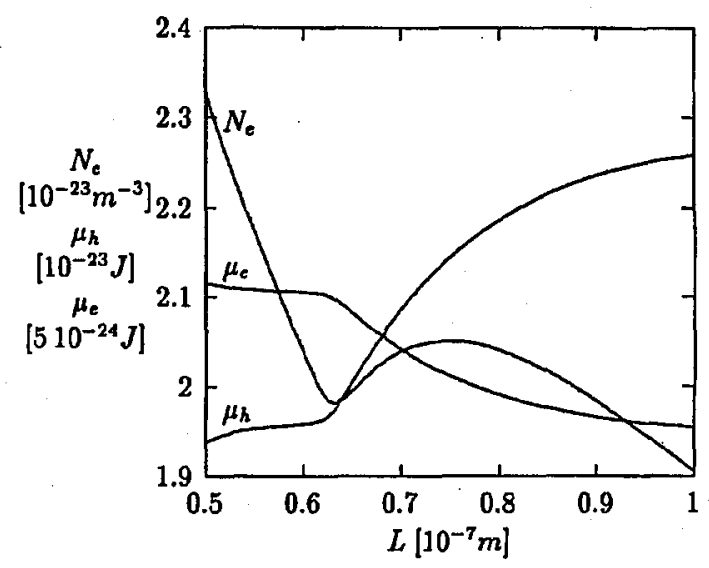

Fig. 1. The dependence of the electron concentration $N_{\mathrm{e}}$, the chemical potential of holes $\mu_{\mathrm{h}}$ and of electrons $\mu_{\mathrm{e}}$ on the thickness $L$ of the thin bismuth film. The values of parameters are: $V_{0}=1000 \times 10^{-22} \mathrm{~J}, w=0.015, b=0.2 \times 10^{-7} \mathrm{~m}, a_{1}=10^{-11} \mathrm{~m}$, $a_{2}=10^{-10} \mathrm{~m}$. The temperature $T=4.2 \mathrm{~K}$.

In Fig. 1 we have also marked the calculated hole and electron chemical potentials. When a thickness of TF decreases the consecutive hole energy levels come below $\mu_{\mathrm{h}}$ which is manifested as small oscillations in the run of the $N_{\mathrm{e}}(L)$ and $\mu_{\mathrm{h}}$, because of a relatively small spacing between these levels. There are $5 \mathrm{such}$ oscillations (at $L=0.539,0.655,0.733,0.820,0.913$ ) and they are hardly seen in Fig. 1. In the interval of the TF thickness which is presented in Fig. 1 there is only one such an oscillation (at $L=0.632$ ) connected with electrons due to the fact that the second level comes below $\mu_{\mathrm{e}}$ when the TF thickness decreases. The 
oscillations in the run of $N_{\mathrm{e}}(L)$ and $\mu_{\mathrm{e}}(L)$ imposed by this fact are much more pronounced because of a relatively big spacing between the electron levels.

Application of the boundary conditions with the potential pockets at the film surfaces enables us to understand the mechanism of the decrease in concentration $N_{\mathrm{e}}$ with increasing thickness $L$. In semi-metals in the potential pocket region the Fermi level is shifted relative to the band edges which lead to increasing local concentration of the one sign carriers and to decreasing concentration of the other sign carriers, but the overlap of the bands remains constant. The total current carrier concentration in the film increases since the density of states increases with increasing energy. With increasing thickness of the film the volume of the near-surface regions becomes a gradually smaller contribution in the total volume of the film which causes a decrease in the concentration $N_{\mathrm{e}}$.

The above analysis indicates that the physical reason for $N_{\mathrm{e}}(L)$ increasing with decreasing $L$ is the presence of hole energy levels deeply inside the near-surface pockets of the potential. The oscillations in the dependence of $N_{\mathrm{e}}(L)$ connected with the passing of subsequent electron levels through the Fermi level explain the details of the run of $N_{\mathrm{e}}(L)$ calculated from experimental data [1,4].

The author wishes to thank Prof. S. Klama for thorough discussions, and Dr. A. Koper for his assistance in writing computer program and helpful discussions. The work has been supported in part by the Committee for Scientific Research via the research grant No. 2 P302 11606.

\section{References}

[1] N. Garcia, Y.H. Kao, M. Strongin, Phys. Rev. B 5, 2029 (1972).

[2] E.A. Bondar', V.I. Vatamanyuk, A.A. Chumak, Thin Solid Films 34, 387 (1976).

[3] H.T. Chu, W. Zhang, J. Phys. Chem. Solids 53, 1059 (1992).

[4] Yu.F. Komnik, Ye.N. Bukhshtab, Yu.V. Nikitin, V.V. Andrievsky, Zh. Eksp. Teor. Fiz. 60, 669 (1971).

[5] G.E. Smith, G.A. Baraff, J.M. Rowell, Phys. Rev. A 135, 1118 (1964).

[6] A.Ya. Shik, Fiz. Tverd. Tela 16, 2801 (1974). 